\title{
The Changes of Nerve Growth Factor Serum Levels After External Muscle Stimulation and The Correlation with Diabetic Neuropathy Symptom in Diabetic Peripheral Neuropathy
}

\author{
Dian Herdiansyah, Marina Annete Moeliono, Tertianto Prabowo \\ Department of Physical Medicine and Rehabilitation, Hasan Sadikin Hospital, Faculty of \\ Medicine, University of Padjajaran Bandung, Indonesia
}

\begin{abstract}
Background: Diabetic Peripheral neuropathy (DPN) is a common complication of diabetes mellitus. Recent studies have demonstrated the involvement of Nerve growth factor (NGF) in the occurrence of DPN. The Diabetes Mellitus was caused reduced the number and disruption of the function of the NGF. External Muscle Stimulation (EMS) might be induced the NGF synthesis. The study objective has to found the effect of EMS on the NGF.

Methods: Study design was before and after treatment without control on subjects with DPN. The blood sample was taken before and after an intervention, as well as the Diabetic Neuropathy symptom (DNSym) and score (DNSc). The EMS treated all of the participants within three times a week for four weeks.

Results: There were 35 subjects were participated. Paired t-test showed a significant increase in NGF serum levels and decrease both DNSym and DNSc with the difference value $12.64 \pm 16.09(\mathrm{p}=0.000), 1.23 \pm 0.82$ $(p=0.000), 1.20 \pm 0.85(p=0.000)$. There was a significant negative correlation between NGF serum level with the DNS- INA $(r=-0.56 ; p=0.001)$ and the DNE-INA $(r=-0.48 ; p=0.007)$.

Conclusion: EMS treatment can increase serum NGF level. EMS has a strong correlation with a decrease in the value of the DNS-INA and DNE-INA.
\end{abstract}

Keywords: Diabetic Peripheral neuropathy, External Muscle Stimulation, Nerve Growth Factor.

\section{Correspondence Detail: \\ Dian Herdiansyah \\ E-mail: dd_herdiansyah@yahoo.co.id}




\section{INTRODUCTION}

Diabetic Peripheral Neuropathy (DPN) is a common complication of diabetes mellitus which affects about one-third of diabetics aged 40 or over with extensive large and small nerve involvement that cause pain, and sensory and motor disturbances. ${ }^{1}$

Recent studies have demonstrated the involvement of Nerve Growth Factor (NGF) in the occurrence of DPN. ${ }^{2}$ In the normal state, NGF helps to regenerate nerve cells by increasing neurofilament synthesis of nerve cells, protects nerves from apoptosis process and as a trigger for $\mathrm{P}$ substance synthesis which functions as a peripheral vascular vasodilator. The condition of diabetes mellitus causes a decrease in the amount and disruption of NGF function. This disorder occurs in diabetes mellitus because of NGF signaling disorder caused by a decrease in the number of NGF receptors, i.e. Trk A and p75 in myelin cells as a result of nerve cell microcirculation disorders. ${ }^{2,3}$

The physiologically human body produces electric fields and current. Changes in the electrical field or action potentials across the cell membrane can trigger cells to transmit signals and excrete cytokines. In wound healing, Electrical processes occur which produces an endogenous electric field that serves as a driver of cell migration to the edge of the injured tissue. In the condition of nutritional and metabolic disturbance in the body, the electrical properties of the tissue will become abnormal. ${ }^{4}$ In addition to the endogenous electric fields, the cell is also responsive to exogenous electrical fields. Exogenous electric fields can be used to regulate and improve cellular function including nerve cells. Research conducted by Chia-Hong-Kao et al. with the provision of electrical stimulation in experimental mice with diabetes mellitus showed increased NGF secretion in nerve cells. ${ }^{5}$

The electrical muscular stimulation (EMS) was one of the electrical stimulation modality can be used in the management of DPN. The role of EMS was created an exogenous electric field to stimulate the process of NGF signaling; the action was as chemotaxis that guided the NGF to damaged nerve cells. ${ }^{4}$ The EMS was induced contraction of the muscles, that stimulate the muscles to secrete cytokines, especially Interleukin 6 (IL-6) in order to induce the synthesis of NGF. ${ }^{6}$

The study aimed to analyze the effect of EMS on the NGF levels, the Diabetic Neuropathy Symptom (DNS) score, the Diabetic Neuropathy Examination (DNE) score, and the correlation between NGF serum level with the DNS and the DNE score. The hypothesizes were the NGF serum level will increase, in contrast with the DNS and the DNE score were decreased after EMS. There was a negative correlation between the NGF serum level with the DNS and DNE score. 


\section{METHODS}

This experimental study was reviewed and approved by the research and ethics review committee of Medical Faculty of Padjadjaran University, Bandung, Indonesia. All research subjects have given the informed consent.

The inclusion criteria of the study were (1) age 40 -60 years, (2) diagnosed with type 2 Diabetes mellitus, (3) the DNS score $\geq 1$ and the DNE score $>3$. (4) Fasting blood glucose level 70 to $125 \mathrm{mg} / \mathrm{dl}$, and 2 hours post-prandial blood glucose level 70 to $200 \mathrm{mg} / \mathrm{dl}$. The exclusion criteria of the study were (1) have contraindication of the EMS, i.e. pacemaker user, heart arrhythmia, or thrombosis or Thrombophlebitis at lower extremity, (2) history of moderate intensity of exercise on the past one month.

Thirty-five participants who met the inclusion criteria were recruited at Polaris club at Ferren clinic Purwakarta. All of the participants were examined to meet the eligibility. The primary outcomes of this study were the NGF serum level, the DNS and DNE score. The NGF serum level was assessed by the ELISA Kit for Nerve Growth Factor. Questionnaires assessed the DNS and DNE (Indonesian version) score.

The blood sample was taken about 5cc a day before the intervention. All of the participants have the EMS treatment on both gastrocnemius muscles. The EMS was by the Chattanooga Intellect Transport Electrotherapy ${ }^{\circledR}$ (DJO Global, USA) for 30 minutes with the biphasic current mode, frequency $70 \mathrm{~Hz}$, pulse duration $0,1 \mathrm{~ms}$. The method of application was alternately repetitive by 3 seconds of rest time, 3 seconds of rising time, and 3 seconds of contraction time. The intensity of the electrical stimulation was adjusted in the range of tolerable level that causing contraction of the muscle and avoiding the pain. The frequency of the EMS was three times a week for four weeks. The NGF serum level, the DNS and the DNE score were re-examined a day after the last treatment.

The data were analyzed by paired t-test to found the effect of the EMS on NGF serum level, the DNS and the DNE score. The Spearman's correlation test analyzed the correlation between the NGF serum level with the DNS and the DNE score. Statistically, significance was determined with p-value $<0.005$. The SPSS version 21.0 for Windows calculated all statistical analysis.

\section{RESULTS}

The study on 30 participants who accomplished all the examination. The characteristics of Participants' was shown in Table 1. 
Table 1. Participant's Characteristics at Baseline

\begin{tabular}{|c|c|c|}
\hline Variable & Mean+SD & $(\%)$ \\
\hline Age (Years) & $54.20 \pm 3.67$ & \\
\hline \multicolumn{3}{|l|}{ Gender } \\
\hline Male & & $7(23.3)$ \\
\hline Female & & $23(76.7)$ \\
\hline BMI $\left(\mathrm{Kg} / \mathrm{m}^{2}\right)$ & $25.56 \pm 2.17$ & \\
\hline
\end{tabular}

* BMI: Body Mass Index

The data in Table 1 has shown the subjects were 23 (76.7\%) female, the aged was $54.2 \pm 3.7$ years, and the

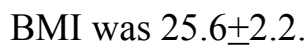

Table 2. The Difference of Nerve Growth Factor Level, DNS and DNE Scores Before and After Intervention

\begin{tabular}{lcccc}
\hline & Before & After & Difference & P Value \\
\hline NGF (pg/dl) & $19.53 \pm 23.39$ & $32.18 \pm 28.24$ & $12.64 \pm 16.09$ & $0.000^{*}$ \\
\hline DNS score & $2.90 \pm 0.60$ & $1.66 \pm 0.71$ & $1.23 \pm 0.82$ & $0.000^{*}$ \\
\hline DNE score & $4.70 \pm 0.70$ & $3.50 \pm 0.62$ & $1.20 \pm 0.85$ & $0.000^{*}$ \\
\hline
\end{tabular}

*Pair t-test

There were no adverse events occurred during EMS treatment. The list of data in Table 2 had described the NGF serum level before EMS was $9.53 \pm 23.39 \mathrm{pg} / \mathrm{dL}$, while after EMS was $32.18 \pm 28.24 \mathrm{pg} / \mathrm{dl}$, with a difference was $12.64 \pm 16.09(\mathrm{p}<0.001)$.

The DNS score before the intervention was $2.90 \pm 0.60$ after the intervention was $1.66 \pm 0.71$. The difference between was $1.23 \pm 0.82(\mathrm{p}<0.001)$. The DNE score before the intervention was $4.70 \pm 0.70$, after an intervention was $3.50 \pm 0.62$, with the difference was $1.20 \pm 0.85(\mathrm{p}<0.001)$.

Table 3. Correlation Difference between Nerve Growth Factor Level with DNS-INA and DNE-INA

\begin{tabular}{llc}
\hline \multicolumn{1}{c}{ Variable } & r & p-Value \\
\hline$\Delta$ NGF $(\mathrm{pg} / \mathrm{dl})-\Delta$ DNS score & -0.56 & $0.001^{*}$ \\
\hline$\Delta$ NGF $(\mathrm{pg} / \mathrm{dl})-\Delta$ DNE score & -0.48 & $0.007^{*}$ \\
\hline
\end{tabular}

*The Spearman's test

The data in Table 3 have shown that there was the moderate negative correlation between the NGF serum level with the DNS and DNE score $(r=-0.56 \mathrm{p}=0.001)$ and $(\mathrm{r}=-0.48 \mathrm{p}=0.007)$. 


\section{DISCUSSION}

This is the only study on the change of the NGF serum levels in subjects with Diabetic Peripheral Neuropathy after EMS in Indonesia. The Previous study by Yu-Jung Chang et al. has found an increase in the NGF levels after the External Stimulation intervention of culture cells. ${ }^{7}$ Another study by Chia-Hong Kao et al. have shown the benefit of the electrical stimulation in diabetes mellitus mice to increase the secretion of NGF serum in nerve cells. ${ }^{5}$

The result of this study has shown a significant increase in the NGF serum levels, although the increase has not reached the normal levels. A study by Keller and Engelhardt has found the aged over 40 years were experienced a $16.6 \%$ decrease in muscle mass and a $40.9 \%$ decrease in muscle strength, and be continued to decrease by $1-2 \%$ each year after 50 year, thus the decreasing of muscle contraction, and the as a consequence was a reduction of the NGF secretion. ${ }^{8,9}$

The study by Jinhee Woo's has revealed that the NGF levels were increased after the intervention by the aerobic exercise. The increased of NGF serum level was lead by the production of cytokines as the production from contraction of large muscles especially in the lower extremities that involved in the aerobic exercise. $^{10}$

Physiological muscle contraction stimulates the secretion of cytokines, especially Interleukin-6 (IL-6), that the intensity and duration of exercise determined the level. The level of the IL- 6 was increasing by the increased of the exercise intensity. ${ }^{11}$ The skeletal muscle secreted the IL- 6 and then induced the synthesis of NGF. ${ }^{6}$

A study by Steensberg et al. has shown that the IL- 6 was secreted in the physical activity on mild intensity (VO2max: 20\%) for 30 minutes, while a study by Soo-Jin Park has found the elevated of the NGF during walking training. ${ }^{12}$

The EMS caused a submaximal contraction of the muscle. However, the previous studies have found that the muscle contraction produced by EMS would be sufficient to induce the secretion of NGF. ${ }^{12}$

Further research is needed to compare the effects of electrical stimulation on the effect of aerobic exercise on levels of NGF serum. All of the subjects were members of Prolanis club connected to a primary clinic, who routinely performed diabetes exercise. To eliminate the bias, thus all of the subjects have not done the diabetes exercise for one month.

The increased NGF serum levels were followed by clinical improvements that could be observed based on the decrease in DNS and DNE scores, that has shown in Table 4.3, Table 4.4, and Table 4.5. The study has found that DNS scores decreased significantly with the difference values of $-1.23 \pm 0.82$ and the DNE with 
a difference of $-1.20 \pm 0.85$. The correlation study has found a negative correlation between the NGF serum levels with the DNS score $(r=0.56 ; p=0.001)$ as well as with the DNE score $(r=0.48 ; p=0.007)$. The resulting study was consistent with the previous study by Humpert et al. that provided 4-week of the EMS therapy on the quadriceps muscle has shown a significant improvement measured using Neuropathy Symptom Scores (NSS) and Neuropathy Disability Scores (NDS) questionnaires. ${ }^{13}$ The two questionnaires were following modified to Diabetic Neuropathy Symptoms (DNS) and Diabetic Neuropathy Examination (DNE).

This study used the Indonesian version of Diabetic Neuropathy Symptoms and Diabetic Neuropathy Examination. The DNS and the DNE validation tests on DPN patients performed by Jayaprakash. The study assumed that the DNS has a high sensitivity but low specificity, while the DNE has low sensitivity but has a high specificity, so the use of DNS with DNE simultaneously will give a high sensitivity and specificity.

The limitation of this study was the variables that could affect the serum levels of NGF and was not taken into account, i.e., the physical activities of subjects related to work and daily activities and the age range of participants was so full; The duration of the study is only four weeks; thus the observation was the shortterm effects of the intervention. The other limitation was not known about how long for the subjects have suffered from Diabetes Mellitus until the development of the DPN.

\section{CONCLUSIONS}

The Electrical Muscular Stimulation has a positive effect on increasing the NGF serum level and reduces sensory symptoms that have shown by the decrease of the DNS and the DNE score. Besides, there was a negative correlation between the NGF serum level with the DNS and the DNE score. According to the results of this study, the DNS and the DNE scores can be used to determine the progression of therapy in patients with DPN.

\section{REFERENCES}

1. Aring M. JD, Falko M. Evaluation and Prevention of Diabetic Neuropathy. AAFP. 2005;71:2123-8.

2. Pittenger Gary VA. Nerve Growth Factor and Diabetic Neuropathy. Experimental Diab Res. 2003; 4:271-85.

3. Kavak Servet TM, Anlar Ömer. Effects of transcutaneous electrical nerve stimulation on a motor and sensorial nerves for diabetic polyneuropathy patients by use of electromyography. Cell Membranes and Free Radical Research. 2010;2.

4. Meng Shiyun RM, Zhang Ze. Electrical Stimulation in Tissue Regeneration: InTech; 2013. Available from: http://www.intechopen.com/books/applied-biomedical-engineering/electrical-stimulation-intissueregeneration. 
5. Kao C-H CJ-J. High-Frequency Electrical Stimulation Can Be a Complementary Therapy to Promote Nerve Regeneration in Diabetic Rats. PLoS ONE. 2013;8.

6. Laudiero Luisa Bracci AL, Caroleo Maria Cristina Endogenous NGF regulates CGRP expression in human monocytes and affects HLA-DR and CD86 expression and IL-10 production. Blood. 2005;106.

7. Chang Yu-Jung HC-M. Electrical stimulation promotes nerve growth factor-induced neurite outgrowth and signaling. Biochim Biophys Acta. 2013;1830:4130-6.

8. Karsten Keller ME. Strength and muscle mass loss with aging process. Age and strength loss. Muscles, Ligaments and Tendons Journal. 2013;4:346-50.

9. Maria RdL. Resistance Training for Muscle Strength and Lean Mass in Adults Older Than 60 Years - A Systematic Review. IJMRPS. 2016;6:16-27.

10. Woo J. The Effects of Exercise on Neurotrophins, Hepatocyte Growth Factor (HGF), and Oxidative Stress in Obese Children. J Life Sci. 2012;22:569-74.

11. Morettini M PM, Sacchetti M, Castiglione F, Mazzà C. A system model of the effects of exercise on plasma Interleukin-6 dynamics in healthy individuals: Role of skeletal muscle and adipose tissue. PLoS ONE. 2017;12(7).

12. Soo-Jin Park M-SY. Effect of exercise on the expression of nerve growth factor in the spinal cord of rats with induced osteoarthritis. J Phys Ther Sci. 2015;27:2551-54.

13. Humpert Per M. MD MMM. External Electric Muscle Stimulation Improves Burning Sensations and Sleeping Disturbances in Patients with Type 2 Diabetes and Symptomatic Neuropathy. Clin J Pain. $2009 ; 10$.

14. Jayaprakash BA. Validation of bedside methods in evaluation of diabetic peripheral neuropathy. Indian J Med Res. 2011;133:645-9. 